\title{
Research and implementation of the service for Batch data with directory import into PLM system
}

\author{
Yanyan Tang ${ }^{1, \mathrm{a}}$, Hong Ji $^{1, \mathrm{~b}}$,Jia GaO ${ }^{1, \mathrm{c}}$, Dexiong $\mathrm{Xu}^{1, \mathrm{~d}}$ \\ ${ }^{1}$ Beijingcomputing centerbeijing 100094 \\ atangyy@bcc.ac.cn, bjihong@bcc.ac.cn, cgaojia@bcc.ac.cn, ${ }^{\mathrm{d}} \mathrm{xudx} @$ bcc.ac.cn
}

Keywords: storage rules, File data import, Rollback, SBDP.

\begin{abstract}
In order to better support applicationof PLM, thedemand thatthe batch of unstructured datawhich according to the defined directory structure to store to PLMisincreasing.In the process of execution, there are many problems such as the large amount of data, the complicated storage directory structure and so on.At present, setting up storage rules and importing the batch data by manual when the PLM in handling this kind of data. So that,this is a complex, error-prone and inefficient process,and the errorsIn the manual operation process is difficult to detect.This paper from 2 aspects to discuss how to implement bulk unstructured data import into PLM. They are the original directory information and document information recognition, the importing interface and complete the batch data import process.
\end{abstract}

\section{Introduction}

PLM - "product life cycle management system", whichintegrate the person, processe, and data informationeffectively. It will be effect the whole enterprise, traverse product from concept to scrap the whole life cycle. Supporting collaboration with product research and development, management, distribution and product definition information.Its core function (such as data warehouse, content management, workflow, document management and project management and task management, etc.). With the gradualapplicationof PLM,Enterprise needs to manage the original data, such as design drawings, photographs, description, and so on. Issues found during use are as follows:

First of all, low efficiency. The efficiency that by manually build complex storage structure and large quantities of data importing into PLM is very low. Second, error-prone.In the process of artificial buildingcomplex storage structureandmass data is error-prone.And not easy to discover in time, because of the large complex structure.Third, at present, The PLM which running in our company is from PTC Windchill.The number of the tools which areintegrated with Windchill system is limited.Many design tools (such as $3 \mathrm{~d}$ Max, Maya, etc.)is not integrated.The project file from the design tools because of the directory structure is too complex to save.Thenon-final results files can be in PLM easily. Through this service, the process file can bulk import to PLM. In order to use in the future, such as version rollback and so on.

To solve these problems, this paper discussed" research and implementation of The service for Batch data with directory into PLM system(SBDP)”.

\section{SBDP}

SBDPis a specific service for PLM. Users only need to specify the local directory and the corresponding fields in PLM system directory, the services which will create directory and copy the documents in the PLM automatically according to the local storage structure and user data document. The purpose of the service is convenient for users. The service development is divided into three parts: the user access, Information identification and data import into PLM, bulk delete.

\subsection{User access}

"User access "--the service will be deployed on the server-side. Bulk operations on system data, so the users must have an administrator permissions can use this service.If and only if all operations are complete, data can be read. "User access", is divided into two steps: first login user password 
authentication; Second, Windchill shell needs to authentice the organization administrator permissions and password.

\subsection{Information identification and data import into PLM}

\subsubsection{Establishstoragerules}

In the process of establishing the storage rule, first,identifying storage rules, then,read the corresponding folder attribute information to generate XML data. Last,establish storage rule in the PLM. As shown in Figure 3.

It traverses thedirectory structureby layerallthe specified locationunder thefolderto generatea recordfor eachfolder, data recording format- "\# SubFolder, user, folderPath, adminDomain, parentContainerPath".Subfoldersgeneratedafter theparentrecord mustdocument in order toavoidthe system generatesstorage directory, the situation could not findthe parent folderappear. Local store directoryas follows:

\begin{tabular}{|c|c|}
\hline $2014-02-11$ & \\
\hline$\Leftrightarrow \Leftrightarrow \nabla 1$. PLM & $4-02 \_11 \mathrm{~V}$ \\
\hline$\triangle$ File & Date \\
\hline D. 142640-BH006002 & $2014 / 3 / 3 \quad 21: 07$ \\
\hline D. 142641-BH006004 & $2014 / 3 / 321: 08$ \\
\hline 142643-BH006042 & $2014 / 3 / 321: 08$ \\
\hline 13. 142644-BH0006057 & $2014 / 3 / 321: 08$ \\
\hline D. 142645-BH006059 & $2014 / 3 / 321: 08$ \\
\hline D. 142646-вH006060 & $2014 / 3 / 3 \quad 21: 06$ \\
\hline
\end{tabular}

Figure 1 Local store directory

Create a csv file according to $<$ Windchill $>\backslash$ LoadFiles $\backslash$ csvmapfile.txt format. Theformatofcsv file as follows:

\# SubFolder, user, folderPath, adminDomain, parentContainerPath

SubFolder ,, / Default / FOLDER1 ,, SubFolder „, / Default / FOLDER1 / SUB01 „,

Data recording

“SubFolder,,/Default/ photo/Original/third/2014_02_11/PLM/PDM/142643-BH006042”,

Recursiveloopin turnall thedata in thedirectoryspecified by the useraregeneratedcorresponding directorystructure information. Txtpreliminarygeneratedas follows:

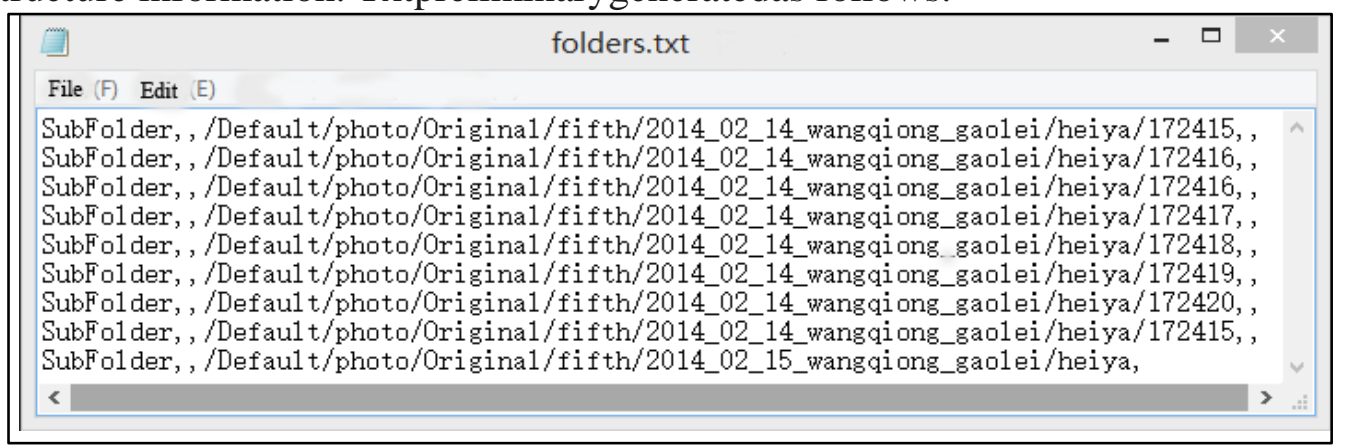

Figure 2 Store directory information

2.generate XML data of folders

Create a folder under loadFilesWindchill $\backslash$ src directory, and this csv file into this folder. Open the Windchill shell, switch to the Windchill \ src \ directory under loadFiles use windchill wt.load.util.CSV2XML test.csv command csv files into xml files.

windchill wt.load.util.CSV2XML -input test0.csv

Note: If there Chinese Note Save the CSV file in UTF-8 encoding format, and use the command windchill wt.load.util.CSV2XML -input test.csv -encoding UTF8

Example xml file as follows: 


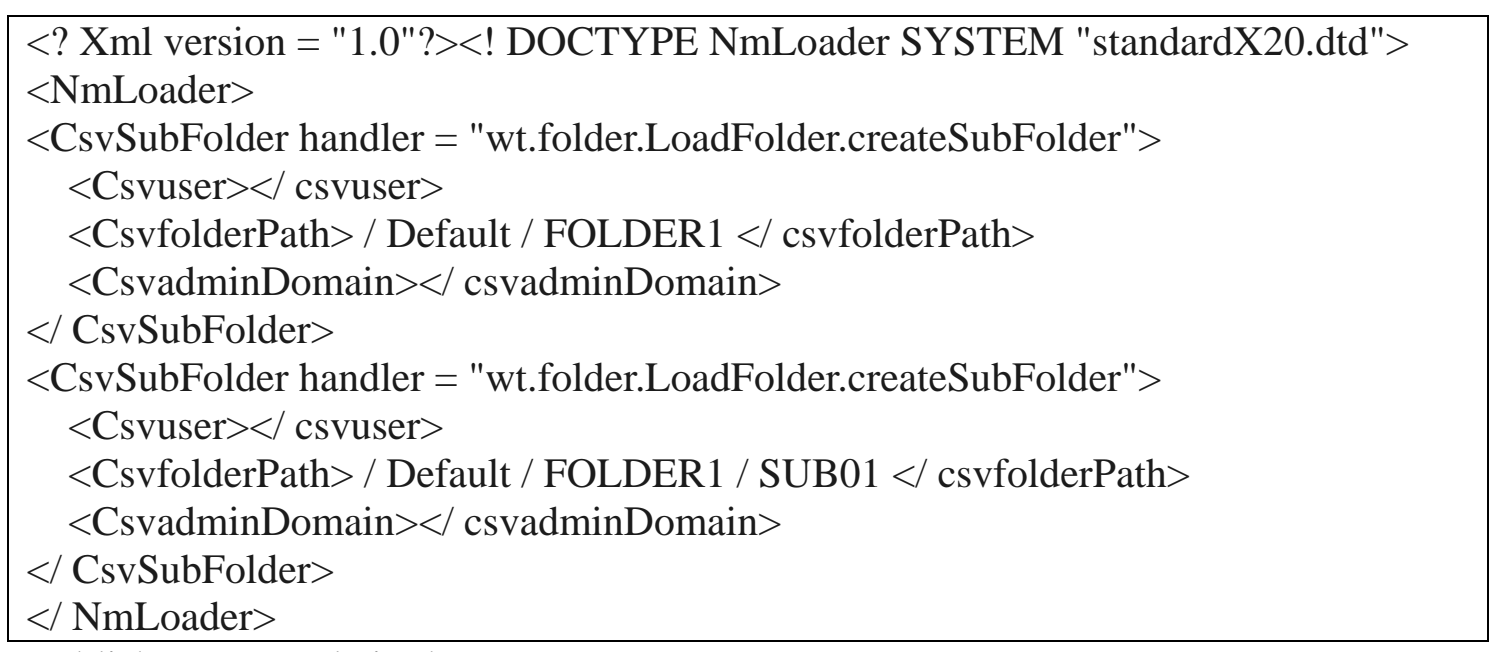

3. Establish storage rule in the PLM

Execute the following command in Wind chill shell folder loading Product or repository. wind chill wt.load.LoadFromFile -d filename.xml -u username -p password -CONT_PATH \"/ wt.inf.container.OrgContainer = organization $/$ wt.pdmlink.PDMLinkProduct $=$ product base $\backslash "$

Table1 Parameters for Establish storage rule in the PLM

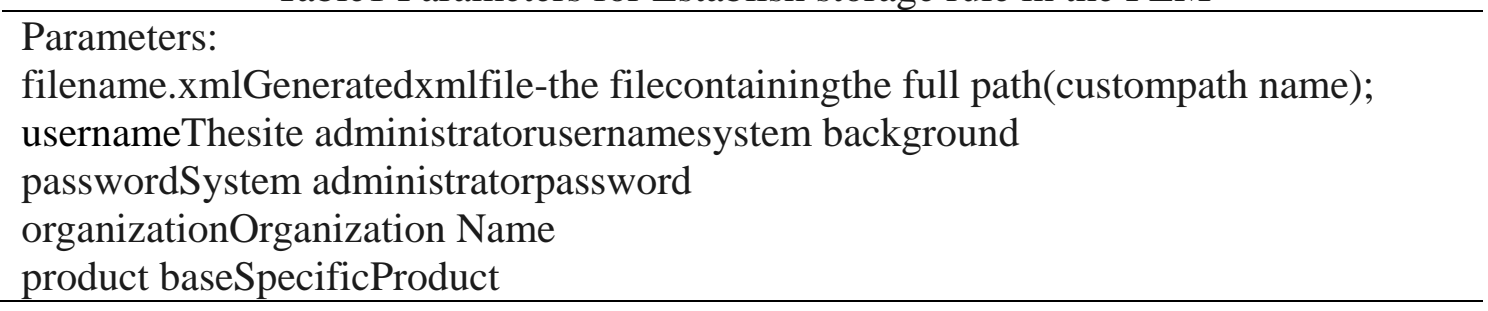

For instance:

windchillwt.load.LoadFromFile -d name1.xml -u wcadmin -p wcadmin -CONT_PATH ।"/wt.inf.container.OrgContainer=BCC/wt.pdmlink.PDMLinkProduct= Natural Museum Digital Project $\backslash "$

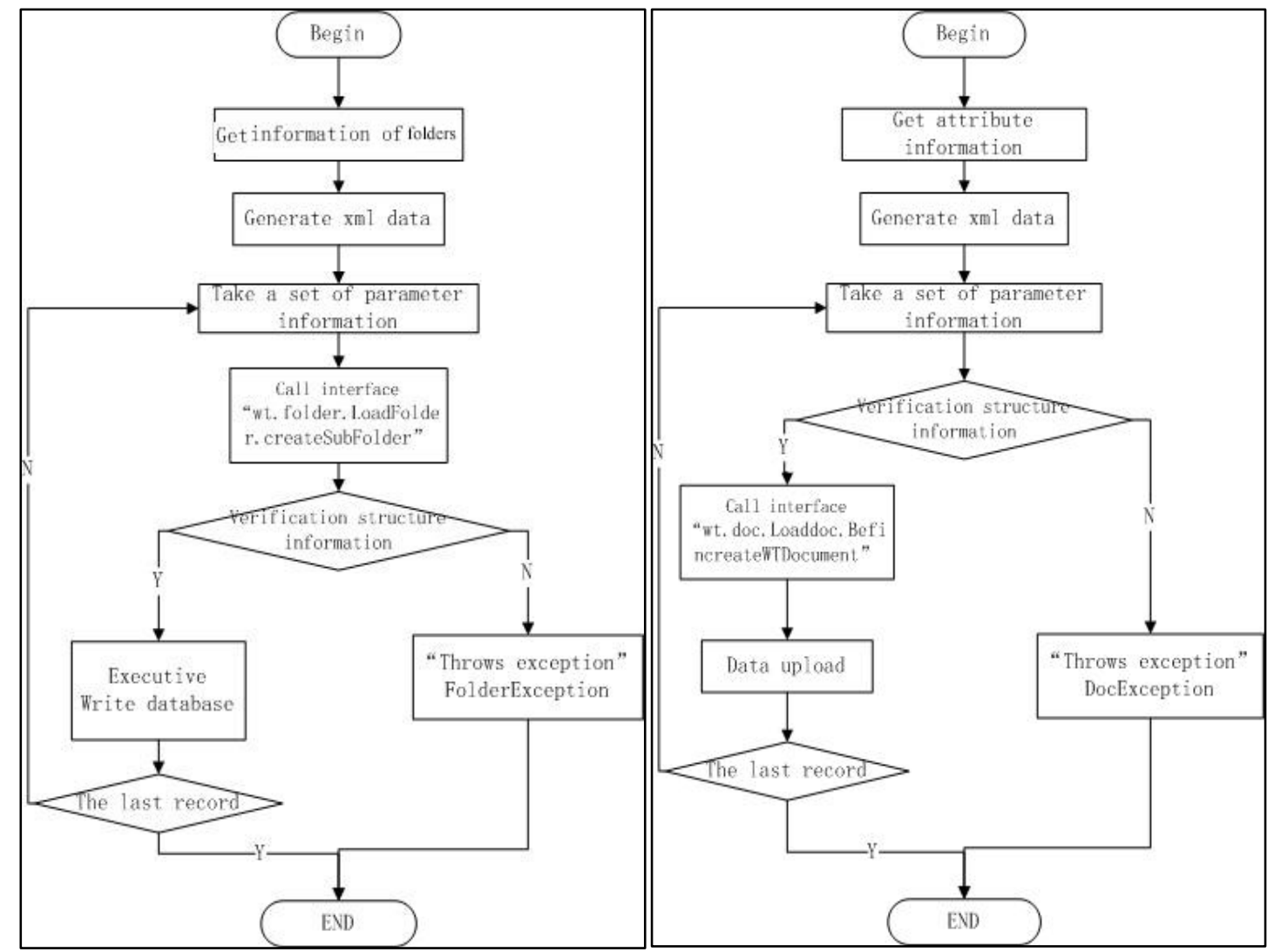

Figure 3 process diagram generated by PLMFigure4 PLM data bulk import the flow chart 


\subsubsection{File data import}

The process of file data import into the system, shown in Figure 4.

1. Identification data file information, Data files are stored locally as shown in figure:

\begin{tabular}{|ll|}
\hline 083102-PP0000855 & \\
\hline File & Date \\
\hline$\square 083102-\mathrm{A}-1 . \mathrm{CR} 2$ & $2014 / 3 / 5 \quad 19: 20$ \\
\hline $083102-\mathrm{A}-1 . \mathrm{JPG}$ & $2014 / 3 / 5 \quad 19: 22$ \\
$0083102-\mathrm{h}-2 . \mathrm{CR} 2$ & $2014 / 3 / 5 \quad 19: 20$ \\
$\square 083102-\mathrm{A}-2 . \mathrm{JPG}$ & $2014 / 3 / 5 \quad 19: 22$ \\
\hline
\end{tabular}

Figure 4 Local file

Recursive loop in turn all the data in the directory specified by the user are generated corresponding node data, as shown in figure:.

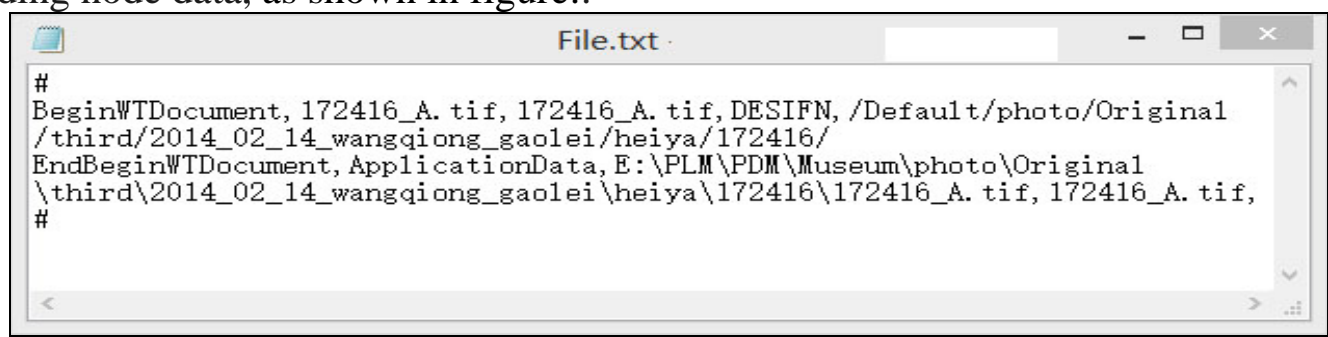

Figure 5 File data information

\section{Generate XML data of data-files}

(1) Call the system background shell-service

Use the following steps:

- Create acsvfileaccording to $<$ Windchill $>\backslash$ LoadFiles $\backslash$ csvmapfile.txtformat.

- Createafolder underloadFilesWindchill $\backslash$ srcdirectory, andthiscsvfile intothis folder.

- Open theWindchillshell, switch to theWindchill \src \directoryunderloadFilesusewindchill wt.load.util.CSV2XML test.csvcommandcsvfiles intoxmlfiles.

windchill wt.load.util.CSV2XML -input test.csv -encoding UTF8.Examplexmlfileas follows:

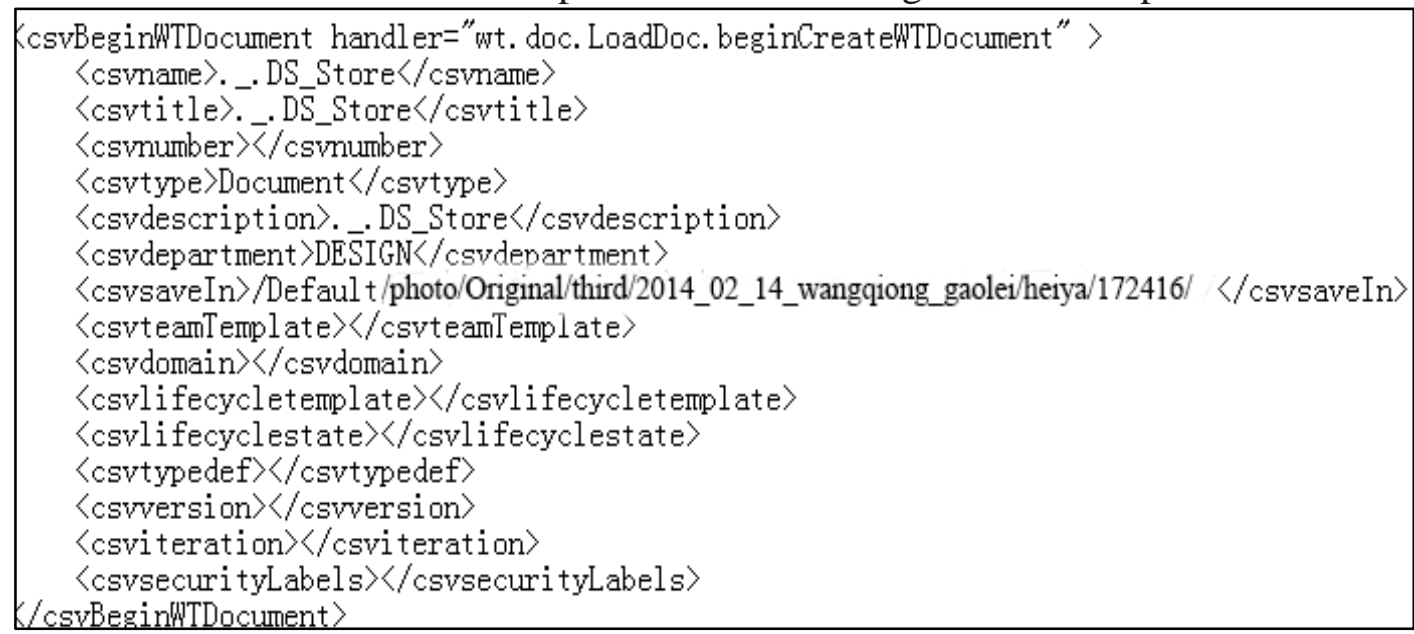

\subsection{Data file import into PLM}

Execute the following command in Wind chill shell folder loading Product or repository.

Wind chill wt.load.LoadFromFile -d filename.xml -u username -p password -CONT_PATH \"/ wt.inf.container.OrgContainer $=$ organization $/$ wt.pdmlink.PDMLinkProduct $=$ product base $\backslash "$ 
Table2 Parameters for File data importinto PLM

Parameters:

filename.xmlgeneratedxmlfile-the filecontainingthe full path(custompath name);

usernameThesite administratorusernamesystem background

passwordsystem administratorpassword

organizationOrganization Name

product baseSpecificProduct

E.g:

Wind chill wt.load.LoadFromFile -d name1.xml -u wcadmin -p wcadmin -CONT_PATH \"/ wt.inf.container.OrgContainer $=$ BCC/wt.pdmlink.PDMLinkProduct =nature museum digital collection!"

Another point to note is that the order of the CSV file, folder handle on the back, to avoid the situation could not find the parent folder, the folder structure that you can sort out first use csv file import.

Results as shown below:

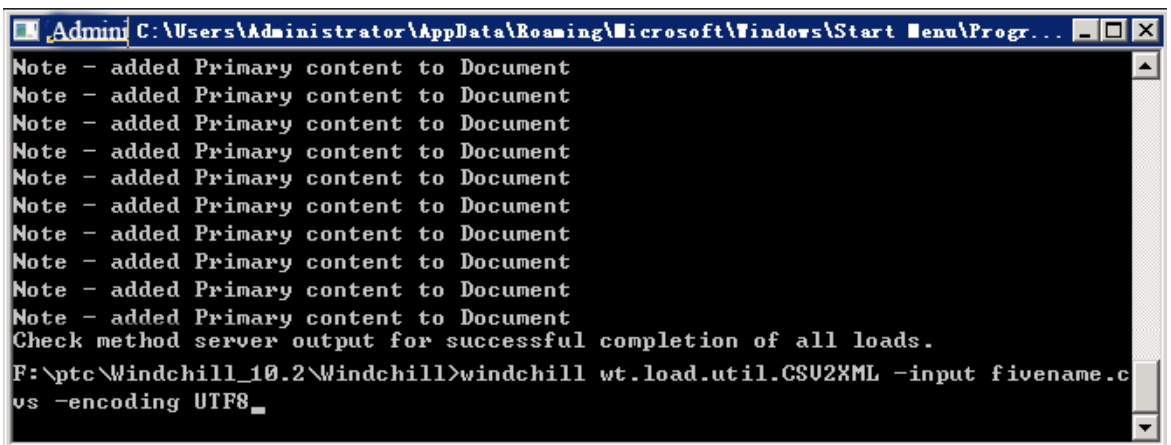

Figure 7 Running on the server

\subsection{Rollback}

For some reason, the system may be back to the state before the operation. In the case of additions and deletions permissions, can be the object of mass delete operation to Realize rollback. Distinguishing all object information specified directory circulating through the system interfaces, batch delete them. Through the client can also complete the batch delete operation. On the client side, choose to delete the directory/file, delete it. System will complete the directory together with the data files in the directory delete, achieve the goal of system rollback.

\section{Summary}

The development of PLM support services (SBDP)not only improve efficiency and reduce the chance of error. The SBDP provides convenient conditions for online sign-trial quantities of data, and management. This is a convenient conditions for the system more widespread application in the future.

\section{References}

[1]. Ji Hong , Chen Liu, Tang YY. Research and implementation of Design Data Management Processing System [J] . Applied Mechanics and Materials Vols.644-650, 2014, pp 2744-2750.

[2]. SU Shao Hui, Integration of CAD \& PDM for DFMC[J].Computer Integrated Manufacturing Systems, 2005(11), 6: $799-804$.

[3]. HAMER P V, LEPOETER K. Managing design data: the five dimensionsof CAD frameworksand configuration management and product data managemen $[\mathrm{J}]$. Proceeding of the IEEE, 1996，8 (4 1): $42-56$. 
[4]. MICHAEL G B, PETER J S. Preparing for PDM [J] . Manufacturing Engineering, 1997, 8: $209-211$.

[5]. ARY - PEKKA H, JUKKA N. Product data management - eXpIoratory study on state - of -the art in one - of - a - kind industry [J] . Computer in Industry, 2001, 44: 251 - 262.

[6]. YU Wan-jun , LIU Da-you ,YANG Bo. J2EE-based Product Lifecycle of PDM System[J] . Computer Applications, Apr.,2004 Vol.24,No.4:139-143

[7]. LI Tao, ZHONG Shi-sheng. Building of workflow and process management model in PDM[J]. JOURNAL OF HARBIN INSTITUTE OF TECHNOLOGY, V01.38, No.6. Jun.2006: 853-855.

[8]. Zhu Haiping, Wang Zhonghao, Li Peigen. Research on Engineering Change Management Based on PDM [J]. Computer Integrated Manufacturing Systems. Vol.9 NO.7 ,Jul .2003: 537-541 